\title{
Cerebral Vessel Enhancement Using Rigid Registration in Three-Dimensional CT Angiography
}

\author{
Helen Hong ${ }^{1}$, Ho Lee ${ }^{2}$, Sung Hyun $\mathrm{Kim}^{3}$, and Yeong Gil Shin²,4 \\ ${ }^{1}$ School of Electrical Engineering and Computer Science BK21: Information Technology, \\ Seoul National University, San 56-1 Shinlim 9-dong Kwanak-gu, Seoul 151-742, Korea \\ hlhong@cse.snu.ac.kr \\ ${ }^{2}$ School of Electrical Engineering and Computer Science, Seoul National University \\ \{holee,yshin\}@cglab.snu.ac.kr \\ ${ }^{3}$ Dept. of Radiology, Seoul National University Bundang Hospital, 300, Gumi-dong, \\ Sungnam-si, Kyunggi-do, Korea \\ kimsungh@radiol.snu.ac.kr \\ ${ }^{4}$ INFINITT Co., Ltd., Taesuk Bld., 275-5 Yangjae-dong Seocho-gu, Seoul 137-934, Korea
}

\begin{abstract}
In this paper, we propose a robust 3D rigid registration technique for detecting cerebral aneurysms, arterial stenosis, and other vascular anomalies in a brain CT angiography. Our method is composed of the following four steps. First, a set of feature points are selected using a 3D edge detection technique within skull base. Second, a locally weighted 3D distance map is constructed for leading our similarity measure to robust convergence on the maximum value. Third, the similarity measure between feature points is evaluated repeatedly by selective cross-correlation. Fourth, bone masking is performed for effectively removing bones. Experimental results show that the performance of our method is very promising compared to conventional methods in the aspects of its visual inspection and robustness. In particular, our method is well applied to vasculature anatomy of patients with an aneurysm in the region of the skull base.
\end{abstract}

\section{Introduction}

Computed tomography (CT) angiography [1-3] is a useful and noninvasive imaging technique for the evaluation of both the intra- and extracranial vasculature. If the injection of a bolus of intravenous contrast material is timely controlled, CT scans can capture the arterial phase in a large volume of patient anatomy. This makes it possible to detect cerebral aneurysms, arterial stenosis, and other vascular anomalies in the intra- and extracranial arteries.

To get an arterial anatomy in three-dimensional views, source images from CT angiography are often displayed using volume rendering techniques [4-5]. A drawback of the volume rendering techniques is that extensive preprocessing is needed for bone removal. Although some software tools are available, the bone removal process is still time-consuming and often incomplete. In addition, separating arteries from bone or perivascular calcification are difficult particularly in the areas where arteries are contiguous with the bone. As a consequence, aneurysms in the region of the skull base are obscured by bone. These difficulties of bone removal limit the clinical application of volume rendering techniques.

A conventional approach of delineating vessels from bone is to subtract enhanced images which taken after injecting a contrast material from nonenhanced images. This 
subtraction technique assumes that tissues surrounding vessels do not change in position or density during exposure. However, even minor patient movement results in severe distortion or artifacts since the contrast between vessels and surrounding tissues is significantly smaller than that between bone and surrounding tissues. Without the proper handling of patient motion artifacts, the clinical application of a subtraction method is very limited. Even though the motion of a patient can be minimized by taking special precautions of either patient, acquisition system, or both [6], artifacts can not be entirely avoided. To reduce patient motion artifacts, we have to use a retrospective image processing techniques.

Several registration techniques have been proposed to reduce patient motion artifacts in brain CT angiography. A common registration method used in radiology is a manual method which translates and rotates images by interactive but tedious and time-consuming user interactions. In addition, this requires the clinical knowledge and experience. Pixel shifting [7] provides a solution in the situation where artifacts have been caused by gross two-dimensional translational motion. In most cases, patient motion is more spatial and complex and cannot be modeled by such a simple transformation. Yeung et al. [8] proposed a 3D feature detector and a 3D image flow computation algorithm for matching feature points between enhanced and nonenhanced images of brain CT angiography. The processing time for finding 3D feature points takes too much time since the interest index of each voxel position is found by comparing variance values in all 13 directions. Venema et al. [9] developed a global matching method using gray value correlation of skull bases in two time interval brain CT angiography. The alignment by minimizing the difference of intensity values of the both skull bases may not be accurate because the same pixel of individual CT scans may have different density. For the same purpose, Kwon et al. [10] proposed a mutual information-based registration. However, the limited information only using the area of skull base for similarity measure does not guarantee the exact alignment.

These registration methods can minimize patient motion artifacts, however, they still need some progress in computational efficiency and robustness for the clear visualization of cerebral vessels in CT angiography. In this paper, we propose a robust $3 \mathrm{D}$ rigid registration technique for the detection of cerebral aneurysms, arterial stenosis, and any other anomalies in brain CT angiography. Our method consists of the following four steps. First, a set of feature points is selected using a 3D edge detection technique within the skull base. Second, a locally weighted 3D distance map is constructed for leading our similarity measure to robust convergence on the maximum value. Third, the similarity measure between feature points is evaluated repeatedly by selective cross-correlation. Fourth, the bone masking process is performed for removing bones. Experimental results show that our method is more robust than the conventional methods.

The organization of the paper is as follows. In Section 2, we discuss how to extract and select feature points in an angiographic image. Then we propose a similarity measure and the optimization process to find exact geometrical relationship between feature points in the enhanced and nonenhanced images. In Section 3, experimental results show how the method accurately and robustly extracts the extra- and intracranial vessels from the brain CT angiography. This paper is concluded with a brief discussion of the results in Section 4. 


\section{3D Rigid Registration}

Fig. 1 shows the pipeline of our method for the registration of enhanced and nonenhanced images. To find exact geometrical relationship between two volumes, one dataset is fixed as mask volume whereas other dataset is defined as contrast volume which taken after injecting a contrast material to mask volume. The contrast volume is moved during the iterative alignment procedure. Interpolating the contrast volume at grid positions of the mask volume is required for each iteration depending on the transformation. Since rigid transformation is enough to align the skull base, we use three translations and three rotations about the $x$-, $y$-, z-axis. After transformation, the mask is slightly widened by means of dilation with 1-pixel to allow partialvolume effects and acceptable small mismatch. Finally, extracted vessels are displayed by a conventional volume rendering technique.

\subsection{Feature Points Selection}

A traditional approach of finding the correspondence between mask and contrast volume of brain CT angiography requires voxel by voxel correspondence test for entire volume. This is computationally expensive and cannot lead to a clinically accepted technique. Since artifacts almost appear in the region where strong edges are present in the subtracted image, we can accelerate the registration procedure by processing voxels belonging to image edge area only instead of all voxels in the volume.

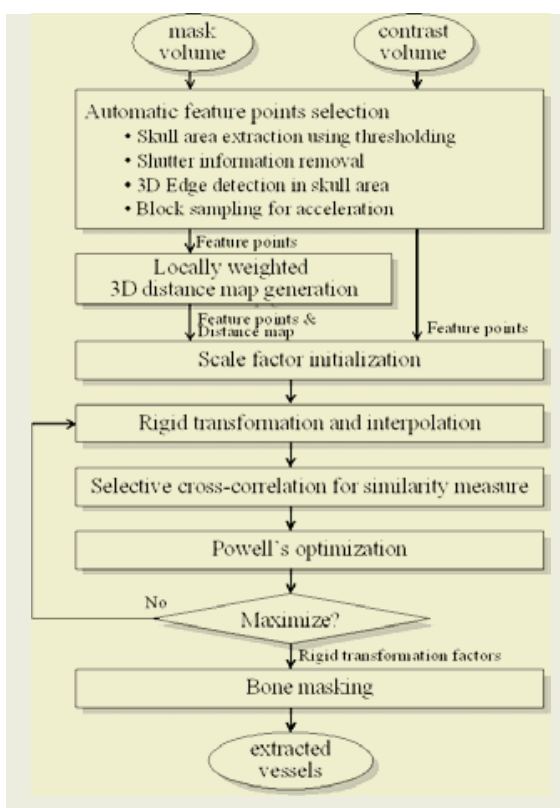

Fig. 1. The pipeline of 3D rigid registration

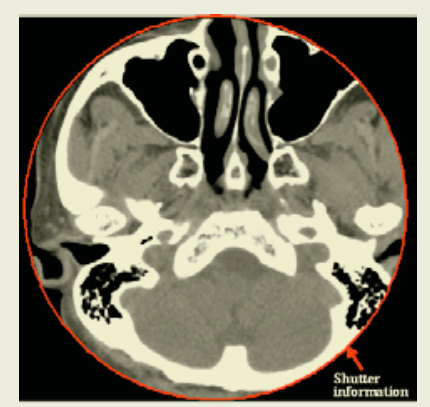

Fig. 2. Shutter information in brain CT angiography image

Our feature identification uses a 3D operator for utilizing spatial relations in volume data. At first, in order to align skull base which represents rigid object boundaries in brain $\mathrm{CT}$ angiography, we only use pixels with a CT number above a chosen threshold. Shutter information of CT angiography shown in Fig. 2 is removed since it leads to misalignment by detecting the boundary of the shutter as a feature. Then $3 \mathrm{D}$ edge detection technique is applied to the skull base in mask and contrast volume, respectively. Just like their two-dimensional counterparts, 3D edges are usually defined as discontinuities of image intensity caused by the transition from one homoge- 
neous $3 \mathrm{D}$ region to another $3 \mathrm{D}$ region of different mean intensity. The location of rigid object boundaries can be computed by detecting local maxima of the gray-level gradient magnitude. Since rigid object boundaries are scale-dependent image features, they can only be detected by using derivatives which allows finer tuning for detecting the required edge scale. Among detected features, algorithm selects a predefined number feature points enough to ensure even distribution of feature points in the skull base.

\subsection{Locally Weighted 3D Distance Map Generation}

Registration between selected feature points is likely to converge to local optimum near to global maximum depending on the initial position. To prevent this occurrence we need to find the optimum value of the similarity measure within the global search space. However, this approach increases the computation time by the proportion to the size of the search space. To minimize the search space and guarantee the convergence to the optimum value, we generate a locally weighted 3D distance map per each feature point. This map is generated only for the mask volume.

For generating 3D distance map, we approximate the global distance computation with repeated propagation of local distances within a small neighborhood mask. To approximate Euclidean distances, we consider 26-neighbor relations for a chessboard distance to be the same distance as 1-distance value. The chessboard distance is applied to each feature point in the mask volume. Then the weighting factor $W_{x y z}$ is multiplied to the chessboard distance as like Eq. (1). The mask size $N$ of locally weighted 3D distance map is fixed as $9 \times 9 \times 9$. Largest weighting factor is assigned to the center of mask while the smallest weighting factor to boundary of mask.

$$
\begin{gathered}
D_{x}=\left|M_{x}-C_{x}\right|, \quad D_{y}=\left|M_{y}-C_{y}\right|, \quad D_{z}=\left|M_{z}-C_{z}\right| \\
W_{x y z}=\frac{(N+1)}{2}-\operatorname{Max}\left(D_{x}, D_{y}, D_{z}\right)
\end{gathered}
$$

where $M$ and $C$ be the current and the center position of locally weighted-3D distance mask. $D_{x}, D_{y}, D_{z}$ is the difference of $x$-, $y$-, and $z$-axis between the current and the center position in locally weighted $3 \mathrm{D}$ distance mask.

Fig. 3(a) shows the locally weighted 3D distance map when $N$ is fixed as $5 \times 5 \times 5$. Fig. 3 (c) shows the cut plane of the volume of a locally weighted $3 \mathrm{D}$ distance map when the distance map of Fig. 3(a) is applied to feature points as shown in Fig. 3(b). Each color shown in Fig. 3(c) represents the distance value of the locally weighted distance map.

\subsection{Similarity Measure and Optimization}

The similarity measure is used to determine the degree of resemblance of windows in successive frames. Several similarity measures have been devised and applied to angiographic images - the sum of squared intensity differences, cross-correlation, and the entropy of the difference image. However, most of these similarity measures are sensitive to mean gray-level offset and local dissimilarities caused by contrasted vessels. We propose the selective cross-correlation as a similarity measure which only 


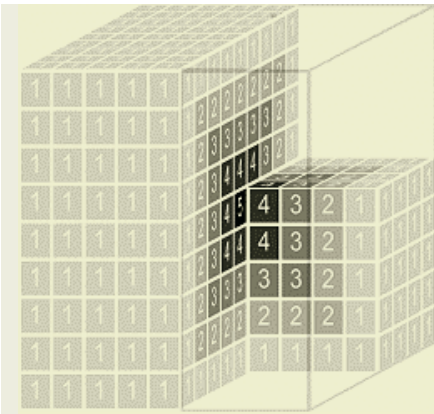

(a)

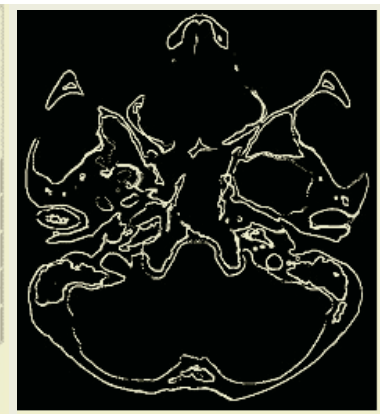

(b)

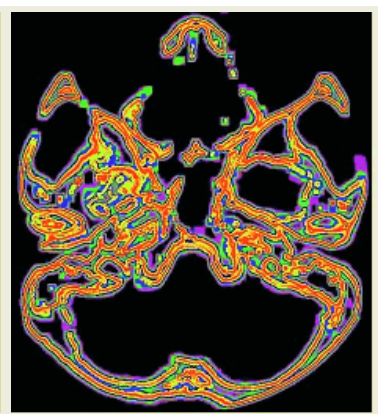

(c)

Fig. 3. A locally weighted 3D distance map

uses feature points near to skull base. Our approach reduces sensitivity to mean graylevel offset or local dissimilarities by incorporating distance information of a locally weighted distance map into the similarity measure.

As can be seen in Eq. (2), the local weighting factor of a 3D distance map in mask volume is multiplied to the distance in contrast volume. We assume that the distances of feature points in contrast volume, $D_{C}(i)$, are all set to 1 . Then the selective crosscorrelation $S C C$ reaches maximum when feature points of mask and contrast volume are aligned correctly.

$$
S C C=\frac{1}{N_{C}} \sum_{i=0}^{N_{C}-1} D_{C}\left(P_{C}(i)\right) W_{M}\left(T\left(P_{C}(i)\right)\right)
$$

where $N_{C}$ is total number of feature points in contrast volume, $P_{C}(i)$ is the position of $i$-th feature point in contrast volume. The weighting factor of the current feature point of contrast volume, $W_{M}(i)$, is obtained from the corresponding locally weighted 3D distance map in mask volume.

To evaluate the selective cross-correlation of large samples from volume dataset, we use the Powell's method. Since the search space of our similarity measure is limited to the surrounding skull base, we do not need a more powerful optimization algorithm such as simulated annealing. For example, Fig. 4 shows the process of optimizing the selective cross-correlation. Fig. 4(a) and (b) are feature points selected from mask and contrast volume, respectively. In the initial position in Fig. 4(c), the number of matching feature points is 6 pixels. Finally, the number of matching feature points becomes 12 pixels when the selective cross-correlation reaches the maximum value.

\subsection{Bone Masking}

A traditional approach for enhancing vessels after alignment is to subtract transformed contrast volume to mask volume. However it is very difficult to remove bone completely using a traditional subtraction technique since densities between mask and transformed contrast volume can be different even in the same pixel position. In addition, partial-volume effects near to bone area and slight amounts of mismatch make it possible to generate artifacts in subtraction images. For the more complete removal of bones, we propose a bone masking instead of a traditional subtraction technique. 
Our bone masking process first identifies bone pixels in the mask volume by applying the threshold value of $1500 \mathrm{HU}$. The identified bone pixels as mask is then slightly widened by means of dilation with 1-pixel to allow for partial-volume effects and slight amount of mismatch. Finally, pixels in contrast volume which are corresponding to pixels in mask volume are set to an arbitrarily low value. This results in the removal of bones in the volume rendering image of contrast volume.

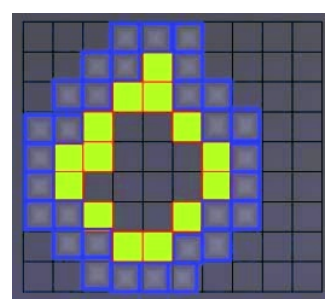

(a)

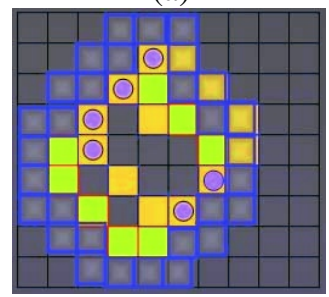

(c)

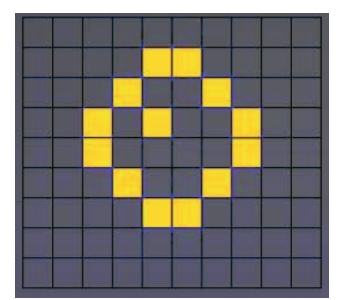

(b)

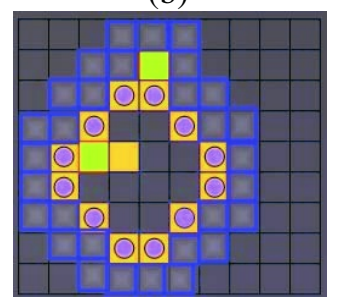

(d)

Fig. 4. The process of optimizing the selective cross-correlation (a) feature points in mask volume (b) feature points in contrast volume (c) initial position (d) final position

\section{Experimental Results}

All our implementation and test were performed on an Intel Pentimum IV PC containing 3.0 GHz CPU and 1.0 GBytes of main memory. Our method has been applied to nine sets of enhanced and nonenhanced images of brain CT angiography, as described in Table 1, obtained from MDCT (Multi-Detector Computed Tomography). We assume that image and pixel sizes are the same in enhanced and nonenhanced images.

Table 1. Experimental datasets

\begin{tabular}{ccccc}
\hline Patient \# & Image size & Slice number & Pixel size & Slice spacing \\
\hline 1 & $512 \times 512$ & 185 & $0.30 \times 0.30$ & 0.3 \\
2 & $512 \times 512$ & 220 & $0.32 \times 0.32$ & 0.3 \\
3 & $512 \times 512$ & 98 & $0.33 \times 0.33$ & 0.7 \\
4 & $512 \times 512$ & 220 & $0.31 \times 0.31$ & 0.3 \\
5 & $512 \times 512$ & 205 & $0.31 \times 0.31$ & 0.3 \\
6 & $512 \times 512$ & 37 & $0.28 \times 0.28$ & 1.0 \\
7 & $512 \times 512$ & 200 & $0.25 \times 0.25$ & 0.3 \\
8 & $512 \times 512$ & 220 & $0.34 \times 0.34$ & 0.3 \\
9 & $512 \times 512$ & 201 & $0.29 \times 0.29$ & 0.3 \\
\hline
\end{tabular}


The performance of our method is evaluated with the aspects of visual inspection and robustness. The average processing time including volume rendering of nine sets is less than 60 seconds. Fig. 5 shows the two-dimensional comparison of a regular subtraction method and the proposed method. We can see many misalignments in Fig. 5 (c), whereas brain vessels are clearly depicted in Fig. 5 (d). Note that intracranial vessels mingling with bone in the middle part of brain are also well separated from skull base. The intracranial vessels in Fig 5 (c) were shrunk by the lack of alignment process of enhanced and nonenhanced images.

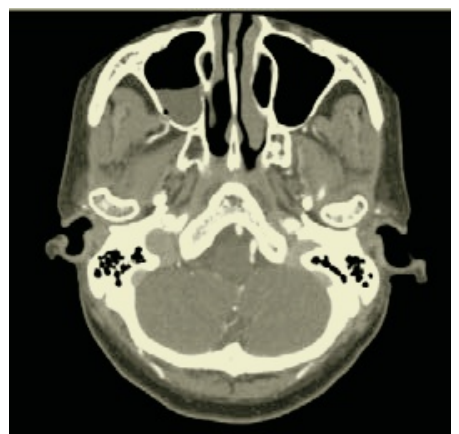

(a)

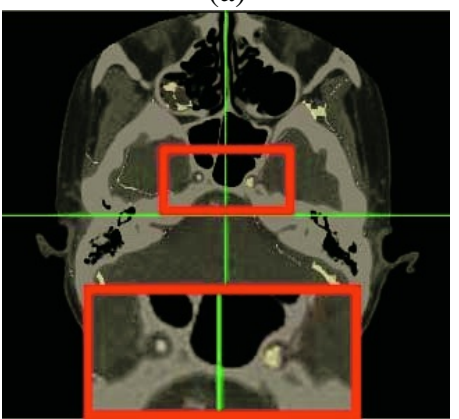

(c)

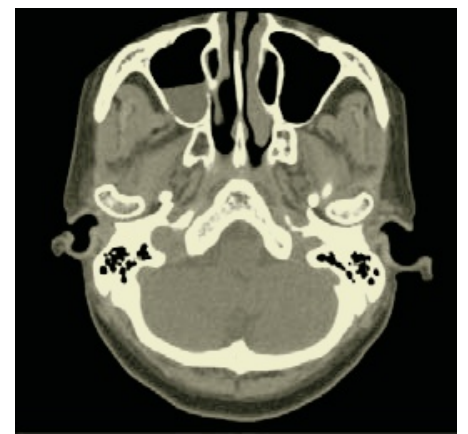

(b)

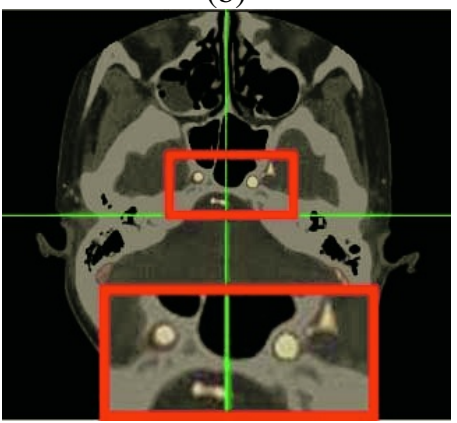

(d)

Fig. 5. Comparison of regular subtraction and the proposed method (a) enhanced image (b) nonenhanced image (c) regular subtraction (d) proposed method

Volumetric images resulted from regular subtraction and the proposed method are illustrated in Fig. 6. Both regular subtraction and the proposed method can reveal the vasculature in the skull base, which are obscured by bone. While the quality of regular subtraction image was substantially reduced by patient motion artifacts, our method can keep the quality of the original image. Our method would be useful for the visualization of the vasculature of the patients with an aneurysm in the region of the skull base.

The robustness of the selective cross-correlation (SCC) criterion has been evaluated by comparing SCC measure traces (represented by circle dot line) with crosscorrelation (CC) measure traces (represented by square dot line). As shown in Fig. 7, the changes of SCC measure are smooth near to the maximal position, but CC measure is changed rapidly. This means that the $\mathrm{CC}$ measure for similarity evaluation is 
more likely to converge to the local maximum, whereas the SCC measure is more robust in converging to the global maximum.

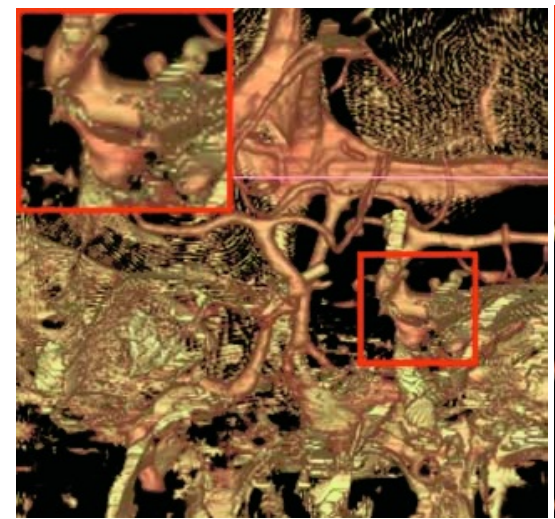

(a)

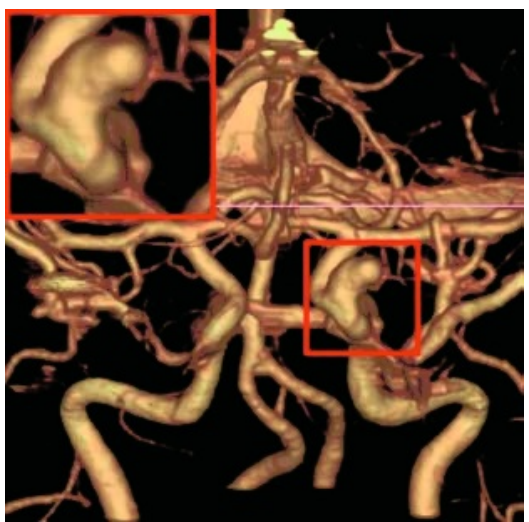

(b)

Fig. 6. Volume rendering images with an ROI of aneurysm in brain CT angiography (a) regular subtraction (b) proposed method

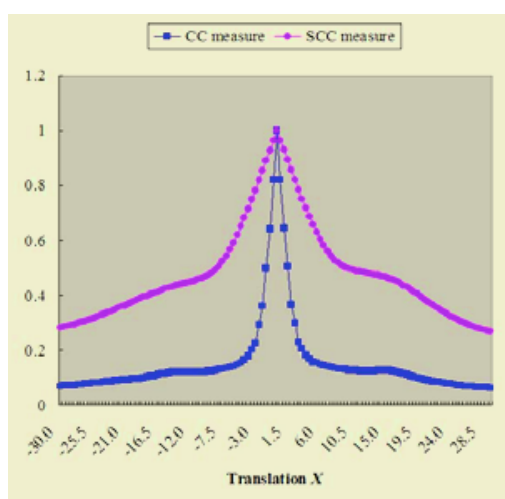

(a)

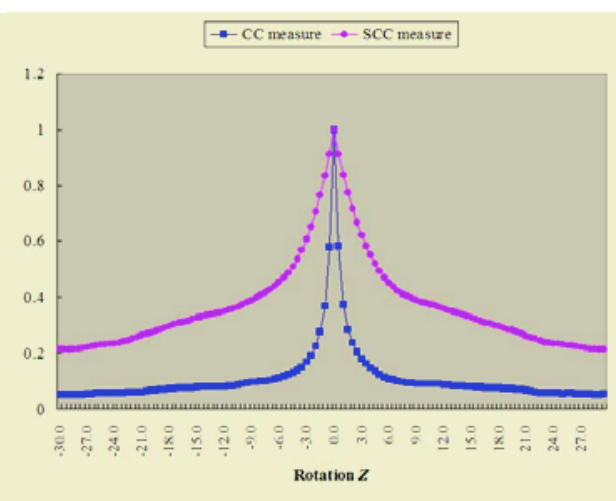

(b)

Fig. 7. Comparison of SCC and CC traces (a) for translation in the $x$-direction (b) for rotation in the $z$-direction.

\section{Conclusion}

To clearly visualize cerebral vessels, we have developed a system for aligning enhanced and nonenhanced images of brain CT angiography. Our methods include 3D feature point selection within the skull base, the generation of a locally weighted $3 \mathrm{D}$ distance map of each feature point, iterative $3 \mathrm{D}$ rigid registration to maximize the similarity measure, the SCC, between feature points, and bone masking. Our method has been successfully applied to the enhanced and nonenhanced brain images of nine different patients. Experimental results show that our registration is clinically useful by the fact that the method is very little influenced by image degradation occurred in 
bone-vessel interface. For all experimental patients, good visualization of cerebral vessels on the volume rendering image can be obtained. In particular, our method has been well applied to vasculature anatomy of patients with an aneurysm in the region of a skull base.

\section{Acknowledgements}

This work was supported in part by the Korea Research Foundation under the Brain Korea 21 Project and INFINITT Co., Ltd. The ICT at Seoul National University provides research facilities for this study.

\section{References}

1. Philip, M.W., Wardlaw, J.M., Easton, V., Can Noninvasive Imaging Accurately Depict Intracranial Aneurysms? A Systematic Review, Radiology, Vol. 217 (2000) 361-370.

2. Kato, Y., Katada, K., Hayakawa, M., Nakane, M., Ogura, Y., Sano, K., Kanno, T., Can 3DCTA Surpass DSA in Diagnosis of Cerebral Aneurysm?, Acta Neurochir (Wien), Vol. 143 (2001) 245-250.

3. Jayaraman, M.V., Mayo-Smith, W.W., Doberstein, C.E., Intracanalicular Aneurysm of the Anterior Inferior Cerebellar Artery Revealed by Multi-Detector CT Angiography, AJNR Am J Neuroradiol, Vol. 24 (2003) 1338-1340.

4. Napel, S., Marks, M.P., Rubin, G.D., Dake, M.D., McDonnell, C.H., Song, S.M., Enzmann, D.R., Jeffrey, R.B., CT Angiography with Spiral CT and Maximum Intensity Projection, Radiology, Vol. 185 (1992) 607-610.

5. Philipp, M.O., Kubin, K., Mang, T., Hormann, M., Metz, V.M., Three-Dimensional Volume Rendering of Multidetector-Row CT Data: Applicable for Emergency Radiology, European Journal of Radiology (2003) 1-6.

6. Jayakrishnan, V.K., White, P.M., Aitken, D., Crane, P., McMahon, A.D., Teasdale, E.M., Subtraction Helical CT Angiography of Intra- and Extracranial Vessels: Technical Considerations and Preliminary Experience, AJNR Am J Neuroradiol, Vol. 24 (2003) 451-455.

7. Wu, Q.X., Bones, P.J., Bates, R.H.T., Translational Motion Compensation for Coronary Angiogram Sequences, IEEE Trans. on Medical Imaging, Vol. 8, No. 3 (1989) 276-282.

8. Yeung, M.M., Yeo, B.L., Liou, S.P., Banihashemi, A., Three-Dimensional Image Registration for Spiral CT Angiography, Proc. of Computer Vision and Pattern Recognition (1994) 423-429.

9. Venema, H.W., Hulsmans, F.J.H., den Heeten, G.J., CT Angiography of the Circle of Willis and Intracranial Internal Carotid Arteries: Maximum Intensity Projection with Matched Mask Bone Elimination - Feasibility Study, Radiology, Vol. 218 (2001) 893-898.

10. Kwon, S.M., Kim, Y.S., Kim, T., Ra, J.B., Novel Digital Subtraction CT Angiography based on 3D Registration and Refinement, Proc. of SPIE Medical Image: Image Processing (2004). 